\title{
Making a choice and sticking with it
}

Mammalian olfactory sensory neurons (OSNs) each express one specific olfactory receptor (OR) throughout their lifespan, and the selected receptor dictates both the response properties of the OSN and its axonal projection patterns. It is thought that a feedback signal, acting through epigenetic silencing mechanisms, prevents the expression of other ORs and stabilizes the expression of the chosen OR; however, the nature of this signal is unknown. Lomvardas and colleagues now reveal a three-node signalling pathway that acts to stabilize the expression of a single OR by an OSN.

OR gene transcription is initiated when the chosen OR allele is 'released' from the pool of silenced OR genes in a process that involves a switch from histone H3K9 methylation (which suppresses transcription) to H3K4 methylation (which activates transcription). The enzyme lysine-specific demethylase 1 (LSD1; also known as KDM1A) can catalyse demethylation of both $\mathrm{H} 3 \mathrm{~K} 9$ and $\mathrm{H} 3 \mathrm{~K}$ 4, suggesting that it could have a role in OR regulation. Indeed, here the authors showed that its expression pattern supports such a role: LSD1 is expressed at high levels in immature OSNs but is rapidly downregulated as the neurons mature after OR expression.
To examine the functional role of LSD1 in OR gene regulation, the authors generated mice in which Lsd 1 was deleted at different stages of OSN development. Removal of Lsd1 before OR expression resulted in a widespread loss of OR gene transcription and caused the premature arrest of OSN development, indicating that LSD1 is involved in desilencing the chosen OR gene and initiating its transcription. By contrast, deletion of $L s d 1$ at later stages had little impact on OR expression.

The authors hypothesized that the downregulation of LSD1 expression after OR selection might be important for the maintenance of OR expression. In support of this idea, they found that mice in which LSD1 was overexpressed in mature OSNs exhibited a marked downregulation of OR expression, which could be restored by suppressing LSD1 expression.

The authors sought to identify factors that might be 'switched on' by OR expression and could regulate LSD1. Adenylyl cyclase type 3 (ADCY3) expression was downregulated in Lsd1-knockout animals but restored when the OR MOR28 (also known as OLFR1507) was overexpressed in these mice, making it a good candidate for such a role. The authors found that deletion of $A d c y 3$ both increased LSD1 expression and delayed maturation of OSNs.
Next, the authors examined the effects of Adcy3 loss on the stability of OR expression using mice in which the gene encoding green fluorescent protein (GFP) was activated by CRE, with CRE being co-expressed with the endogenous Mor28 allele. In these animals, expression of both GFP and CRE by a neuron indicates stable MOR28 expression, whereas expression of either GFP or CRE alone indicates that gene switching has occurred or that OR expression is not stable enough to activate the GFP reporter, respectively. The authors found that gene switching, indicative of unstable OR expression, was higher in Adcy3-knockout mice. These findings suggest that $\mathrm{ADCY} 3$ stabilizes OR expression by downregulating LSD1 and preventing gene switching.

This study has revealed that expression of ADCY3, driven by OR choice, acts as a feedback signal that stabilizes OR expression by downregulating LSD1 and thus preventing release of further OR alleles from epigenetic silencing.

Katherine Whalley

ORIGINAL RESEARCH PAPER Lyons, D. B. et al.

An epigenetic trap stabilizes singular olfactory receptor expression. Cell 154, 325-336 (2013) 